\title{
RMetS
}

\section{Linear theory of the sea breeze in a thermal wind}

\author{
Philippe Drobinski, ${ }^{\text {a*}}$ Richard Rotunno ${ }^{\mathrm{b} \dagger}$ and Thomas Dubos ${ }^{\mathrm{a}}$ \\ a Institut Pierre Simon Laplace/Laboratoire de Météorologie Dynamique, Palaiseau, France \\ ${ }^{\mathrm{b}}$ National Center for Atmospheric Research, Boulder, Colorado, USA \\ ${ }^{*}$ Correspondence to: P. Drobinski, IPSL/LMD, Ecole Polytechnique, Palaiseau 91128, France. \\ E-mail: philippe.drobinski@lmd.polytechnique.fr \\ ${ }^{\dagger}$ The contribution of this author to this article was prepared as part of his official duties as a US Federal Government \\ employee.
}

This article investigates the linear dynamics of the sea breeze in an along-shore thermal wind shear. The present analysis shows that the sea-breeze circulation is tilted towards the slanted isentropes associated with the thermal wind. At a critical value of the thermal wind shear, the tilt of the sea-breeze circulation becomes equal to the slope of the background isentropes. The present analysis also shows a spatial shift between the heating pattern and the sea-breeze circulation. The present linear theory is then applied to interpret measurements made in the vicinity of New York City where there is a warm-season synoptic southwesterly jet. It is compared with observations and past numerical simulations. Agreement is found with respect to the enhanced along-coast wind that follows the tilted isentropes, the order of magnitude of the isentrope tilt and the clockwise rotating wind hodograph showing the jet maximum peaking at $\mathbf{1 8 0 0}$ solar time. There is a disagreement between theory and observations on the phase lag between the jet maximum and the cross-shore pressure gradient maximum. However, this disagreement can reasonably be attributed to either the angle made by the synoptic jet to the coastline and/or the presence of friction. The inland spatial shift of the breeze indicated by the theory might also be indirectly confirmed by the coastal inland wind observations of a larger diurnal amplitude for a stronger synoptic jet. Copyright (c) 2011 Royal Meteorological Society

Key Words: coastal low-level jet; breeze circulation; linear model

Received 8 November 2010; Revised 17 March 2011; Accepted 20 April 2011; Published online in Wiley Online Library 20 May 2011

Citation: Drobinski P, Rotunno R, Dubos T. 2011. Linear theory of the sea breeze in a thermal wind. Q. J. R. Meteorol. Soc. 137: 1602-1609. DOI:10.1002/qj.847

\section{Introduction}

The mesoscale structure of the sea-breeze circulation has in the past been idealized as the response of a rotating, stratified fluid to a diurnally varying differential surface heating (e.g. Walsh, 1974; Rotunno, 1983; Niino, 1987; Dalu and Pielke, 1989; Baldi et al., 2008; Qian et al., 2009; Drobinski and Dubos, 2009). These studies have been useful for the extraction of some basic qualitative features of the theoretical sea-breeze circulation, such as its aspect ratio, phase relative to the diurnal heating cycle, and modification by friction. An aspect that has received relatively less attention is the effect of the summertime land-sea temperature contrast (thermal wind) on the diurnally varying sea-breeze circulation. In this article we report on a simple extension to the theory of Rotunno (1983; R83 hereafter) to include a base-state thermal wind.

The effect of an onshore/offshore background wind on the dynamics of the sea breeze has been heavily investigated through numerical simulation (e.g. Estoque, 1962; Walsh, 
1974; Pielke 1974; Bastin and Drobinski, 2006; Bastin et al., 2006) and observations (e.g. Bastin et al., 2005, 2006; Drobinski et al., 2006, 2007) and only recently through analytical techniques (Qian et al., 2009). The effect of an along-shore thermal wind has received comparatively little attention. Burk and Thompson (1996) modelled the summertime thermally balanced coastal jet on the west coast of the USA. They found that the diurnal sea-breeze circulation is superimposed on the summertime northerly coastal jet and is strongly influenced by elevated and variable coastal terrain as well as relatively cold coastal sea-surface temperatures. Recently a similar phenomenon has been identified on the east coast of the USA (although without the strong topographic effects). Colle and Novak (2010; $\mathrm{CN} 10$ hereafter) find that there is a warm-season southerly jet in the coastal regions of New York and New Jersey that often dislays a strong diurnal modulation. These jet winds are vertically confined to the boundary layer $(<300 \mathrm{~m})$ and display a seasonal maximum occurrence in June-July suggesting that summertime land-sea temperature contrast is important to the jet occurrence. The jet events exhibit a diurnal cycle, rotating in a clockwise sense on a wind hodograph. However, compared to CN10 observations, the effect is much more obvious along the US west coast where the northerly jet and depressed marine layer is a quasipermanent feature of the summertime flow. The coastline is straighter as well.

In the present article we present a simple theoretical description of the above-described phenomena through a modification of the theory for the two-dimensional seabreeze circulation proposed in R83. Using a simple coordinate transformation to geostrophic coordinates (Hoskins and Bretherton 1972), the solutions found in R83 can, for the simple case of spatially constant along-coast wind shear, be used directly. Upon transformation of that solution back to fixed Cartesian coordinates, the effects of constant along-coast shear on the sea breeze can be easily deduced.

In section 2 we introduce the linear model, coordinate transform and solution with its salient features described. Section 3 illustrates the solution for prescribed heating. In section 4 we compare the latter features with the modelled and observed features of the sea-breeze circulations embedded in the coastal jets of the west coast of the USA. Section 5 concludes the study.

\section{The linear model}

\subsection{Equations of motion}

Consider the Cartesian coordinate system shown in Figure 1 with the coastline at the origin and with the dark and light shadings representing sea and land, respectively. For the purposes of the present simple exposition, we consider the horizontally uniform, constant shear, along-coast jet

$$
V=V_{0}-\Lambda z
$$

where $V_{0}$ is a constant and $\Lambda$ is the vertical wind shear. Assuming thermal-wind balance we have

$$
f \frac{\partial V}{\partial z}=\frac{\partial B}{\partial x}
$$

where the base-state buoyancy $B(x, z) \equiv g \Theta / \Theta_{0}, g$ is the gravitational acceleration, $\Theta(x, z)$ is the base-state

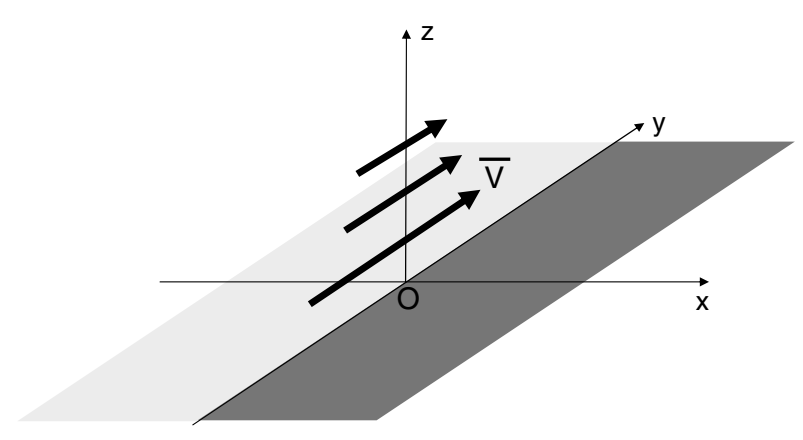

Figure 1. The dark and light shadings represent the cooler sea and the warmer land, respectively. The thermal wind is illustrated by the arrows indicating decreasing southerly wind with height.

potential temperature and $\Theta_{0}$ is a constant reference value. Substituting Eq. (1) into Eq. (2) we have

$$
\frac{\partial B}{\partial x}=-f \Lambda
$$

so that

$$
B=N^{2} z-f \Lambda x+B(0,0),
$$

where $N$ is the Brunt frequency (assumed constant) and $B(0,0)$ is a constant.

The equations of motion linearized about the base-state wind $V(z)$ are

$$
\begin{aligned}
\frac{\partial u}{\partial t}-f v & =-\frac{\partial \phi}{\partial x}+F_{u}, \\
\frac{\partial v}{\partial t}-\Lambda w+f u & =F_{v}, \\
\frac{\partial w}{\partial t}-b & =-\frac{\partial \phi}{\partial z}+F_{w}, \\
\frac{\partial b}{\partial t}+N^{2} w-f \Lambda u & =Q+F_{b}, \\
\frac{\partial u}{\partial x}+\frac{\partial w}{\partial z} & =0,
\end{aligned}
$$

where $(u, v, w)$ are the components of the perturbation wind vector in the directions $(x, y, z)$, respectively, $\phi=p / \rho_{0}$ is the perturbation pressure divided by a reference air density, $b$ is the perturbation buoyancy and $\left(F_{u}, F_{v}, F_{w}, F_{b}\right)$ are friction terms which will first be taken equal to zero. The boundary conditions are $w=0$ at $z=0$ and the requirement for bounded solutions as $x^{2}+z^{2} \rightarrow \infty$. In the following, we consider $N$ to be constant and prescribe $Q=Q(x, z, t)$.

From Eq. (5), one can derive a single equation for the streamfunction $\psi(u=\partial \psi / \partial z$ and $w=-\partial \psi / \partial x)$ :

$$
\left(\frac{\partial^{2}}{\partial t^{2}}+N^{2}\right) \frac{\partial^{2} \psi}{\partial x^{2}}+\left(\frac{\partial^{2}}{\partial t^{2}}+f^{2}\right) \frac{\partial^{2} \psi}{\partial z^{2}}+2 f \Lambda \frac{\partial^{2} \psi}{\partial x \partial z}=-\frac{\partial Q}{\partial x} .
$$

Herein we take $Q=H(x, z) \sin \omega t$ (where $\omega=2 \pi / 24 \mathrm{~h}$ is the diurnal frequency and $t=0$ corresponds to sunrise), so that $\psi \sim \sin \omega t$. Since $N \simeq 10^{-2} \mathrm{~s}^{-1}$ and $\omega \simeq 0.73 \times 10^{-4} \mathrm{~s}^{-1}, N^{2} \gg \omega^{2}$; under the latter conditions, Eq. (6) becomes

$$
N^{2} \frac{\partial^{2} \psi}{\partial x^{2}}+\left(f^{2}-\omega^{2}\right) \frac{\partial^{2} \psi}{\partial z^{2}}+2 f \Lambda \frac{\partial^{2} \psi}{\partial x \partial z}=-\frac{\partial Q}{\partial x} .
$$


Following R83, we set

$$
H=\frac{Q_{\max }}{\pi}\left\{\frac{\pi}{2}-\tan ^{-1}\left(\frac{x}{x_{0}}\right)\right\} \exp \left(-\frac{z}{z_{0}}\right),
$$

where $Q_{\max }, x_{0}, z_{0}$ are the amplitude, horizontal and vertical scales of the heating, respectively. When $x_{0} \rightarrow 0, Q(x, z, t)$ is a step function in $x$ that decays exponentially away from the lower boundary, similar to the heating function used in Drobinski and Dubos (2009).

\subsection{Non-dimensional equations}

Non-dimensionalizing the governing equation (7) with

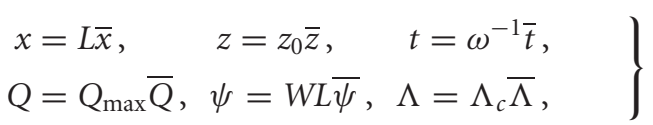

where

$$
\Lambda_{c}^{2} \equiv \frac{N^{2}\left(f^{2}-\omega^{2}\right)}{f^{2}}, L \equiv \frac{z_{0} N}{\sqrt{f^{2}-\omega^{2}}} \text { and } W \equiv \frac{Q_{\max }}{N^{2}},
$$

we have

$$
\frac{\partial^{2} \bar{\psi}}{\partial \bar{x}^{2}}+2 \bar{\Lambda} \frac{\partial^{2} \bar{\psi}}{\partial \bar{x} \partial \bar{z}}+\frac{\partial^{2} \bar{\psi}}{\partial \bar{z}^{2}}=-\frac{\partial \bar{Q}}{\partial \bar{x}}
$$

Without the heating term, Eq. (10) is the familiar equation for symmetric instability (e.g. Bluestein, 1993, p. 317) with instability occurring if $\bar{\Lambda}^{2}>1$. In the present application, we consider the stable case $\bar{\Lambda}^{2}<1$ and situations in which $f>\omega$ (latitude $>30^{\circ}$ ); with the latter restrictions, Eq. (10) is elliptic and may be transformed into

$$
\frac{\partial^{2} \bar{\psi}}{\partial \xi^{2}}+\frac{\partial^{2} \bar{\psi}}{\partial \zeta^{2}}=-\frac{1}{\sqrt{1-\bar{\Lambda}^{2}}} \frac{\partial \bar{Q}}{\partial \xi}
$$

where

$$
\xi=\frac{\bar{x}-\bar{\Lambda} \bar{z}}{\sqrt{1-\bar{\Lambda}^{2}}}, \quad \zeta=\bar{z}
$$

The coordinate transformation (12) is well known in the theory of frontal circulations, as are the solution techniques for the Poisson equation (11) (e.g. Hakim and Keyser, 2001). Here we note that Eq. (11) is identical to (14) of R83 with $\beta=1 / \sqrt{1-\bar{\Lambda}^{2}}$ and thus the solutions given in R83 may be used directly.

\subsection{Solutions}

The solution of Eq. (11) for the point source $-4 \pi \delta\left(\xi-\xi^{\prime}\right) \delta\left(\zeta-\zeta^{\prime}\right) \sin \bar{t}$ far away from boundaries is the Green's function (omitting the time dependence)

$$
G_{\infty}\left(\xi-\xi^{\prime}, \zeta-\zeta^{\prime}\right)=-\ln \left\{\left(\xi-\xi^{\prime}\right)^{2}+\left(\zeta-\zeta^{\prime}\right)^{2}\right\}
$$

(Morse and Feshbach, 1953, p. 798). With the source located at $\xi^{\prime}=\zeta^{\prime}=0$, the dimensional form of the argument of the logarithm in (13) is proportional to

$$
\alpha x^{2}-2 \gamma x z+z^{2}
$$

where $\alpha \equiv\left(f^{2}-\omega^{2}\right) / N^{2}$ and $\gamma \equiv \Lambda f / N^{2}\left(=(\partial z / \partial x)_{B}\right.$ in the light of Eq. (4)). With a rotation of coordinates, (14) can be expressed as

$$
\begin{aligned}
\left(\alpha \cos ^{2} \epsilon\right. & \left.-0.5 \gamma \sin \epsilon+\sin ^{2} \epsilon\right) \widehat{x}^{2} \\
& +\left(\alpha \sin ^{2} \epsilon+0.5 \gamma \cos \epsilon+\cos ^{2} \epsilon\right) \widehat{z}^{2}
\end{aligned}
$$

where

$$
\tan (2 \epsilon)=\frac{2 \gamma}{1-\alpha}
$$

For typical values of the meteorological parameters, $(\alpha, \gamma) \ll 1$; consequently Eq. (16) indicates that $\epsilon \simeq \gamma$ and Eq. (15) reduces to $\alpha \widehat{x}^{2}+\widehat{z}^{2}$. Thus we deduce that surfaces of constant $\psi$ are ellipses, with the ratio of major to minor axes being $N\left(f^{2}-\omega^{2}\right)^{-1 / 2}$ (as in (19) of R83), but tilt so that the major axes align with the tilt of the isentropes $\Lambda f / N^{2}$. The horizontal scale of the land/sea-breeze cell $z_{0} \alpha^{1 / 2}$ is thus comparable to the Rossby radius $z_{0} N / f$ (Drobinski and Dubos, 2009).

The solution of Eq. (11) for the two point sources $-4 \pi \delta\left(\xi-\xi^{\prime}\right) \delta\left(\zeta \pm \zeta^{\prime}\right) \sin \bar{t}$, which satisfies the lower boundary condition $\bar{\psi}(\xi, \zeta=0, \bar{t})=0$, is the modified Green's function (again omitting the time dependence)

$$
G_{0}\left(\xi-\xi^{\prime}, \zeta-\zeta^{\prime}\right)=-\ln \left[\frac{\left(\xi-\xi^{\prime}\right)^{2}+\left(\zeta-\zeta^{\prime}\right)^{2}}{\left(\xi-\xi^{\prime}\right)^{2}+\left(\zeta+\zeta^{\prime}\right)^{2}}\right]
$$

To help investigate the spatial dependence of the solution, we express Eq. (17) in terms of the coordinates $(\bar{x}, \bar{z})$ and consider a point source located at the coast $\bar{x}=0$ and at some level $\bar{z}=\bar{z}_{s}$; thus the image source is located at $\left(\xi^{\prime}, \zeta^{\prime}\right)=\left(-\left(1-\bar{\Lambda}^{2}\right)^{-1 / 2} \bar{\Lambda} \bar{z}_{s}, \bar{z}_{s}\right)$; with the latter specifications, the right-hand side of Eq. (17) becomes

$$
-\ln \left[\frac{\left\{\bar{x}-\bar{\Lambda}\left(\bar{z}-\bar{z}_{s}\right)\right\}^{2}+\left(1-\bar{\Lambda}^{2}\right)\left(\bar{z}-\bar{z}_{s}\right)^{2}}{\left\{\bar{x}-\bar{\Lambda}\left(\bar{z}-\bar{z}_{s}\right)\right\}^{2}+\left(1-\bar{\Lambda}^{2}\right)\left(\bar{z}+\bar{z}_{s}\right)^{2}}\right] .
$$

Figure 2 displays the Green's function $G_{0}$ for a source located at $\bar{z}_{s}=1$ for $\bar{\Lambda}=0$ and $\bar{\Lambda}=0.9$. Several features may be deduced directly from the formulae. First, we note that $G_{0}$ is symmetric with $\bar{\Lambda}=0$ and tilted for $\bar{\Lambda}=0.9$, as expected from the solution for the point source discussed above. Second, we see that the horizontal decay away from the (tilted) maximum is more rapid in the case with $\bar{\Lambda}=0.9$. To deduce the latter analytically, we fix the height $\bar{z}=\bar{z}_{s}$ so that (18) becomes

$$
\ln \left\{1+4\left(1-\bar{\Lambda}^{2}\right)\left(\frac{\bar{z}_{s}}{\bar{x}}\right)^{2}\right\} \simeq 4\left(1-\bar{\Lambda}^{2}\right)\left(\frac{\bar{z}_{s}}{\bar{x}}\right)^{2}
$$

The horizontal scale of $G_{0}$ is therefore proportional to $\sqrt{1-\bar{\Lambda}^{2}}$ in $(\bar{x}, \bar{z})$ coordinates. One can similarly examine the vertical decay for fixed $\bar{x}$; setting $\bar{x}=0$, one can show that $G_{0} \sim \bar{\Lambda} \bar{z}_{s} / \bar{z}$. However, when the Green's function is applied to a given heating function (see below), the vertical scale of the sea-breeze cell cannot be smaller than the vertical scale of the heating itself, and therefore the vertical scale of the sea-breeze cell will be $\sim 1$, independent of $\bar{\Lambda}$. 

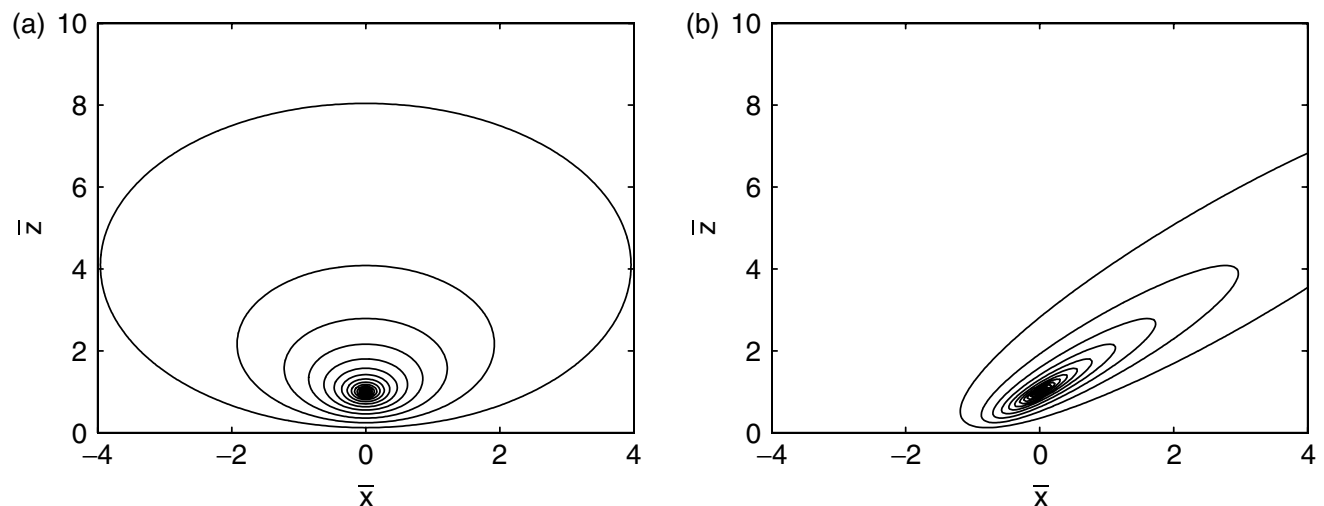

Figure 2. Green's function (Eq. (18)) with point source located at $(0,1)$ for (a) $\bar{\Lambda}=0$ and (b) $\bar{\Lambda}=0.9$.

One further feature that will be useful for later interpretation is the calculation of the cross-coast velocity $\partial \bar{\psi} / \partial \bar{z}$ at $\bar{z}=0$. From Eq. (18) we deduce

$$
\left.\frac{\partial G_{0}}{\partial \bar{z}}\right|_{\bar{z}=0}=\frac{-4 \bar{z}_{s}}{\bar{x}^{2}+2 \bar{\Lambda} \bar{x} \bar{z}_{s}+\bar{z}_{s}^{2}},
$$

which shows that, for $\bar{\Lambda}>0$, the maximum cross-coast wind at the ground moves inland by the distance $-\bar{\Lambda} \bar{z}_{s}$.

\section{Solutions for prescribed heating}

The solution to Eq. (11) for the heating function (Eq. (8)) is given by (24) of R83, which in the present notation is

$$
\begin{aligned}
\bar{\psi}(\xi, \zeta, \bar{t})=-\frac{\beta \sin \bar{t}}{4 \pi} \iint & \ln \left[\frac{\left(\xi-\xi^{\prime}\right)^{2}+\left(\zeta-\zeta^{\prime}\right)^{2}}{\left(\xi-\xi^{\prime}\right)^{2}+\left(\zeta+\zeta^{\prime}\right)^{2}}\right] \\
\times & \frac{\partial \bar{H}\left(\xi^{\prime}, \zeta^{\prime}\right)}{\partial \xi^{\prime}} \mathrm{d} \xi^{\prime} \mathrm{d} \zeta^{\prime}
\end{aligned}
$$

which in $(\bar{x}, \bar{z})$ coordinates becomes, using Eq. (8),

$$
\begin{aligned}
& \bar{\psi}(\bar{x}, \bar{z}, \bar{t})= \\
& \frac{\beta \sin \bar{t}}{4 \pi} \iint \ln \left[\frac{\left\{\bar{x}-\bar{x}^{\prime}-\bar{\Lambda}\left(\bar{z}-\bar{z}^{\prime}\right)\right\}^{2}+\left(1-\bar{\Lambda}^{2}\right)\left(\bar{z}-\bar{z}^{\prime}\right)^{2}}{\left\{\bar{x}-\bar{x}^{\prime}-\bar{\Lambda}\left(\bar{z}-\bar{z}^{\prime}\right)\right\}^{2}+\left(1-\bar{\Lambda}^{2}\right)\left(\bar{z}+\bar{z}^{\prime}\right)^{2}}\right] \\
& \times \frac{1}{\pi} \frac{\bar{x}_{0} \exp \left(-\bar{z}^{\prime}\right)}{\bar{x}_{0}^{2}+\bar{x}^{2}} \mathrm{~d} \bar{x} \mathrm{~d} \bar{z}
\end{aligned}
$$

The solutions for $\bar{\psi}$ for $\bar{\Lambda}=0,0.5$ and 0.9 are shown in Figures 3 and 4; the zero-shear case reproduces the solution shown in figure $2 \mathrm{a}$ of R83. In the presence of shear, the linear response displays an asymmetric shape of the breeze cell with a seaward tilt $(x>0)$, which increases with the shear $\left(\sim \Lambda f / N^{2}\right)$. Onshore $(x<0)$, the sea-breeze cell seems to be compressed to a shallower depth and to extend farther inland than in the absence of shear. However the seabreeze aspect ratio is maintained independent of the shear, as shown previously by the scaling analysis. The intensity of the breeze also increases with shear.

The sea-breeze strength and direction as a function of time, visualized by hodographs, is a key aspect of the seabreeze dynamics. Figure 5 shows the wind hodographs (polar diagram where wind direction is indicated by the angle from the centre axis and its strength by the distance from the centre), rotating clockwise from the south at $\bar{t}=0$ (sunrise), as a function of distance to the shore and for various values of the shear $\bar{\Lambda}$. At a fixed height $(\bar{z}=0.1$ in Figure 5$)$, the present solutions indicate that the sea breeze is weaker offshore for stronger shear and the magnitude of the diurnal wind variation decreases with increasing shear, whereas the opposite behaviour is found onshore. Indeed, onshore $(x<0)$, Figures 5(a) and (b) show that the magnitude of the wind fluctuations is larger in the presence of shear. Offshore, the sea breeze tilt implies that near-surface wind decreases rapidly for increasing distance from the shore. Since the tilt increases with shear, despite the intensification of the sea breeze with shear, for a given distance from the shore and a given height, the near-surface wind decreases with increasing shear. Onshore, the absence of tilt and the increase of the breeze intensity with shear implies that, for a given distance from the shore and a given height, the near-surface wind increases with increasing shear.

\subsection{Spatial lag between heating and breeze cell}

Equation (20) indicates a spatial shift between the heating pattern and the sea-breeze response. Figure 6(a) displays the horizontal wind component $\bar{u}$ close to the surface. It shows that the $\bar{u}$ disturbance is shifted onshore as $\bar{\Lambda}$ increases (see also Figure 3 ) and that the magnitude of the nearsurface wind increases with increasing shear onshore while it decreases with increasing shear offshore.

Figure $6(\mathrm{~b})$ shows the position $\bar{x}_{\max }$ of the near-surface wind maximum as a function of $\bar{\Lambda}$. Consistent with Eq. (20) implying that $\bar{x}_{\max } \sim-\bar{\Lambda} \bar{z}_{s}$, Figure 6 (b) shows evidence of a linear relationship between $\bar{x}_{\max }$ and $\bar{\Lambda}$.

\section{Similarities to and differences from observations}

In CN10's climatological study, the southwesterly nearsurface jet that develops primarily during the warm season east of the northern New Jersey coast and south of Long Island ranges typically between 11 and $17 \mathrm{~m} \mathrm{~s}^{-1}$ (Figures 2 and 3 of $\mathrm{CN} 10$ ). The wind directions for the jet (Figure 6 of CN10) trace out a nearly elliptical orbit for the $24 \mathrm{~h}$ period, similar to the inertial rotation of the sea breeze (e.g. Neumann, 1984). CN10 discusses a geostrophic adjustment during the day for the near-surface jet, so by the end of the day the flow is quasi-balanced on a larger scale. The scale of the adjustment is relatively large (Rossby radius), so the enhanced winds extend well offshore (Figures 13d 

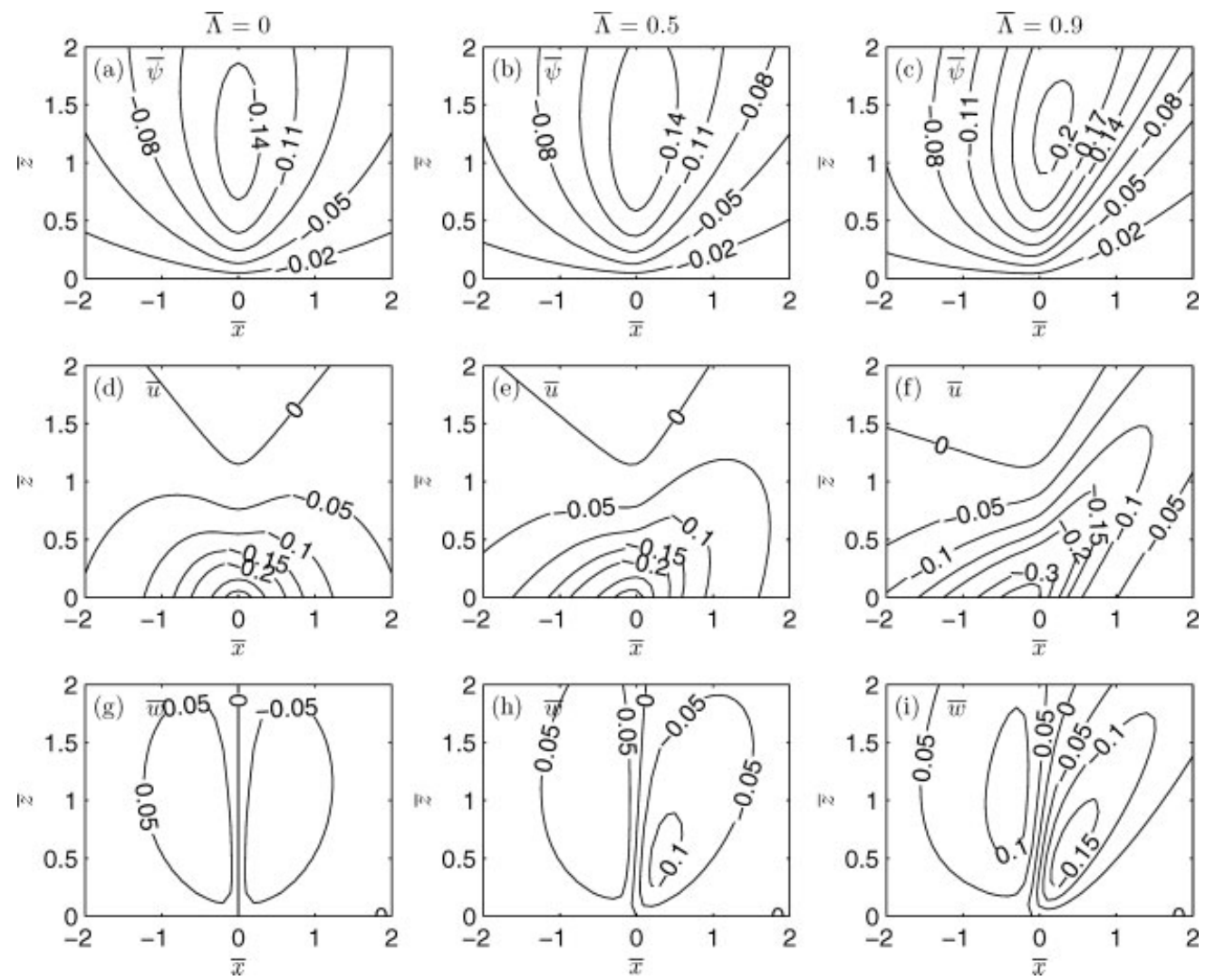

Figure 3. Upper, middle and lower rows display the non-dimensional streamfunction $\bar{\psi}(\bar{x}, \bar{z}), \bar{u}$ and $\bar{w}$ wind components, respectively, at $\bar{t}=\pi / 2$. Left, centre and right columns are for $\bar{\Lambda}=0,0.5$ and 0.9 , respectively.
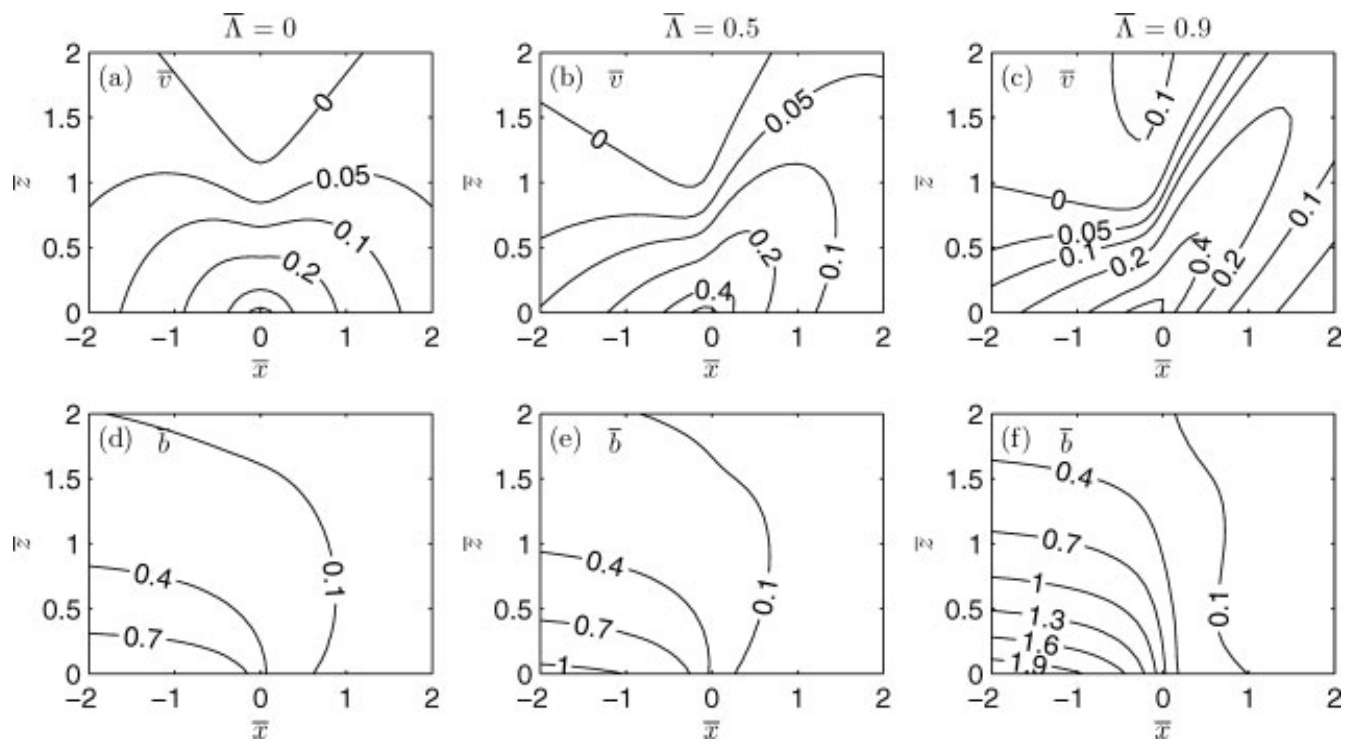

Figure 4. Upper and lower rows display the non-dimensional $v$ and $b$ wind components, respectively, at $\bar{t}=\pi$. Left, centre and right columns are for $\bar{\Lambda}=0,0.5$ and 0.9 , respectively.

and 21a of CN10). This is also consistent with linear seabreeze theory which shows that the horizontal scale of the land/sea-breeze cell is comparable to the Rossby radius (Drobinski and Dubos, 2009). Thus our interpretation is that the near-surface jet described in $\mathrm{CN10}$ might be the combination of a synoptically induced thermal wind and a sea breeze, and the large number of similarities with seabreeze dynamics (diurnal modulation, inertial rotation, scale of the adjustment of the order of the Rossby radius, etc.) make us confident that such a concept might be relevant.
In CN10, the jet maximum is reached at about 1800 local solar time (LST) (Figures 4 to 6 of CN10) and is most probably a combination of the ambient southwesterlies forced at synoptic scales, with $V_{0} \sim 5-10 \mathrm{~m} \mathrm{~s}^{-1}$, and the more local sea breeze, typically $\sim 5 \mathrm{~m} \mathrm{~s}^{-1}$. The time of the jet maximum occurs $2-5 \mathrm{~h}$ after the maximum land-sea temperature difference or pressure contrast (Figure 7 of CN10). In CN10's numerical investigation of one particular case, the enhanced along-coast wind basically follows the tilted isentropes similarly to Figures 3 and 4 . The value 
of the isentrope tilt ranges between about 2 and $5 \times 10^{-3}$ (Figures 15 and 16 of CN10). The vertical shear of the horizontal wind $\Lambda \sim 4-5 \times 10^{-3} \mathrm{~s}^{-1}$ (Figures 13 and 14 of $\mathrm{CN} 10$ ), and the thermal stratification $N \sim 10^{-2} \mathrm{~s}^{-1}$ (tephigrams in Figures 11, 15 and 16 of $\mathrm{CN} 10$ ). For the region surrounding New York City, $f \sim 9.5 \times 10^{-5} \mathrm{~s}^{-1}$, so the scaling variables are

$$
\begin{aligned}
\Lambda_{c} & \equiv \frac{N}{f} \sqrt{f^{2}-\omega^{2}}=6.4 \times 10^{-3} \mathrm{~s}^{-1} \\
\text { and } L & \equiv \frac{z_{0} N}{\sqrt{f^{2}-\omega^{2}}} \sim 50 \mathrm{~km}
\end{aligned}
$$

with $z_{0} \sim 0.3 \mathrm{~km}$, therefore $\bar{\Lambda} \sim 0.6-0.8$. In our model, the tilt of the isentropes is $\Lambda f / N^{2} \sim 6.0 \times 10^{-3} \mathrm{~s}^{-1}$, which is larger than the tilt simulated in CN10 (between 2 and $5 \times 10^{-3}$ ), but still within an acceptable range considering the simplicity of our linear approach.

There is also a good qualitative comparison with the wind hodographs (Figures 6 and 12 in CN10) (Figure 5). Indeed, the wind hodographs in $\mathrm{CN} 10$ display a clockwise rotation in agreement with the present theory. The jet maximum is reached at $\sim 1800 \mathrm{LST}$, which in non-dimensional time would be $\bar{t}=\pi$. In our model, $\bar{u}$ is in advance and $\pi / 2$ (i.e. $6 \mathrm{~h}$ ) out of phase with $\bar{\phi}$. Conversely $\bar{v}$ and $\bar{\phi}$ are in phase, so that at sunrise and sunset $(\bar{t}=0$ and $\bar{t}=\pi)$, the breeze blows parallel to the coast with high pressure to the right. The jet maximum is $\sqrt{u^{2}+(V+v)^{2}}$. If $V \gg(u, v)$, then the jet maximum is obtained when the synoptic wind jet blows in the same direction as the $\bar{v}$-component of the breeze, i.e. from the southwest. This corresponds to high pressure over the sea at $\bar{t}=\pi$, consistent with the CN10 observations. The effect of the shear, $\bar{\Lambda}$, on the hodograph cannot be assessed quantitatively. Qualitatively, the wind measurements in CN10 are collected inland near the shore. The climatological hodograph (Figure 6 in CN10) corresponds to a jet maximum of about $10 \mathrm{~m} \mathrm{~s}^{-1}$, whereas the hodograph of the case-study (Figure 12 in CN10) corresponds to a jet maximum of about $15 \mathrm{~m} \mathrm{~s}^{-1}$. We cannot directly infer the value of the wind shear $\bar{\Lambda}$ (except for the CN10 numerical simulation where $\bar{\Lambda} \sim 0.6-0.8$ ), but we can postulate that the stronger the jet maximum, the stronger the shear. The climatological hodograph displays typical diurnal wind variation of about $7 \mathrm{~m} \mathrm{~s}^{-1}$ (between 5 and $12 \mathrm{~m} \mathrm{~s}^{-1}$; Figure 6 in CN10), whereas the magnitude of the variations reach $10 \mathrm{~m} \mathrm{~s}^{-1}$ for the strong jet case-study (between 2 and $12 \mathrm{~m} \mathrm{~s}^{-1}$; Figure 12 in CN10). This difference, also predicted theoretically in Figure 5(b), might be attributed to the spatial shift of the sea breeze inland and the seabreeze intensification with shear onshore, as discussed in the previous section. Even though this interpretation must be taken with care (because of measurement uncertainties, typically of the order of $1 \mathrm{~m} \mathrm{~s}^{-1}$ ), the behaviour of the observed hodographs might be an indirect validation of the inland shift of the breeze in the presence of a superimposed synoptic along-shore jet.

Although the similarities between CN10 observations and our linear model reveal a broad agreement with respect to some of the basic flow features, our simple model assumptions limit a more quantitative one-to-one correspondence. Indeed, there are significant differences between CN10 observations and our model:
- the observed/modelled evolution of the near-surface jet is tightly correlated with the evolution of a meridional pressure gradient, which cannot be included in the 2D linear model;

- the observed jet maximum occurs about 2 to $5 \mathrm{~h}$ later than the pressure gradient maximum, while they are in phase in the linear model.

Regarding this last aspect, in our model, $\bar{v}$ and $\partial \phi / \partial x$ are in phase, so that the pressure gradient maximum should be found when $\bar{v}$ is maximum (i.e. at sunset, $\bar{t}=\pi$ ). In $\mathrm{CN} 10$, the jet maximum occurs about 2 to $5 \mathrm{~h}$ later than the pressure gradient maximum. So, the phase lag between the jet maximum and the cross-shore pressure or temperature gradient is apparently not consistent with our theory. The explanation for this difference could be twofold: (i) the absence of a cross-shore wind which can interact with the sea-breeze, and (ii) the absence of friction in our model which is known to play a major role on the phasing between the sea-breeze cell and surface heating (R83). The effect of a cross-shore background wind is geometrical, and results from the fact that the wind speed is a maximum when $U_{0} u+V_{0} v$ is a maximum if $U_{0} \neq 0$. Taking for $u$ and $v$ the values given by the current theory with $U_{0}=0$, and since they are then in quadrature, one finds a phase lag $\chi$ given by $\chi=\tan ^{-1} U_{0}|u| / V_{0}|v|$. Considering a $10 \mathrm{~m} \mathrm{~s}^{-1}$ offshore synoptic jet making an angle of $45^{\circ}$ with the shore to the left, one finds a jet maximum occurring about $2 \mathrm{~h} 45 \mathrm{~min}$ after the maximum cross-shore pressure gradient, which is not inconsistent with $\mathrm{CN} 10$, considering the shape of the concave coast line where the cross-shore pressure gradient as well as the wind components are measured. Accounting for friction, by adding a linear friction term (R83; Drobinski and Dubos, 2009), i.e. $\left(F_{u}, F_{v}, F_{w}, F_{b}\right)=-v(u, v, w, b)$ (where $v$ is a friction coefficient), is equivalent to the transformation $\partial / \partial t \rightarrow \mathcal{L}=\partial / \partial t+v$. To investigate the effect of friction, we compute the circulation budget of the breeze flow:

$$
C=\int_{0}^{+\infty}\{u(x, 0, t)-u(x,+\infty, t)\} \mathrm{d} x .
$$

The vertical branch may be neglected in the hydrostatic approximation. The evolution of $C$ is obtained from Eqs (5) and (23), so

$$
\begin{aligned}
\mathcal{L} C= & \underbrace{f \int_{0}^{+\infty}\{v(x, 0, t)-v(x,+\infty, t)\} \mathrm{d} x}_{F V} \\
& \underbrace{-\int_{0}^{+\infty}\{b(-\infty, z, t)-b(+\infty, z, t)\} \mathrm{d} z}_{P G}
\end{aligned}
$$

where $F V, P G$ are the Coriolis and pressure gradient contributions. The pressure gradient term $P G$ is directly related to the buoyancy through the hydrostatic approximation ( $P G=B$ in R83 and Drobinski and Dubos, 2009). Following the methodology of R83, one can easily show that the circulation budget is not affected by the presence of the thermal wind-induced shear $\Lambda$, and is the same as in R83 (which is not surprising since Eq. (11) is identical to Eq. (14) of R83). We can thus show that the breeze circulation and heating display a phase lag

$$
\chi_{1}=\tan ^{-1}\left\{\frac{2 v \omega}{f^{2}+v^{2}-\omega^{2}}\right\}
$$


(for $f^{2}+v^{2}>\omega^{2}$ since $\left.f>\omega\right)$ (R83). Similarly, the phase lag between buoyancy or pressure gradient and heating is $\chi_{2}=\tan ^{-1}(\omega / \nu)(\mathrm{R} 83)$. The Coriolis term $F V$ thus displays a phase lag $\chi_{1}$ with respect to the pressure gradient $P G, F V$ being a maximum after the pressure gradient maximum is reached. Figure 7 displays phase lag $\chi_{1}$ of the Coriolis force relative to pressure gradient as a function of the linear friction parameter $v$ for $f=10^{-4} s^{-1}$. It effectively shows that friction induces a lag between $F V$ and $P G$, and thus between $v$ and the pressure gradient $\partial \phi / \partial x$. Figure 7 also shows that $\chi_{1}$ quickly tends to a value of about $\pi / 4$, i.e. $3 \mathrm{~h}$, which is close to the value observed by $\mathrm{CN} 10$. At this stage, it is difficult to conclude which is the dominant process that can explain the phase lag between the pressure gradient maximum and the jet maximum. A full nonlinear modelling system in an idealized framework would be more suited to address this issue.

\section{Conclusion}

This article investigates the linear dynamics of the sea breeze in a thermal wind. It shows that the breeze cell modified
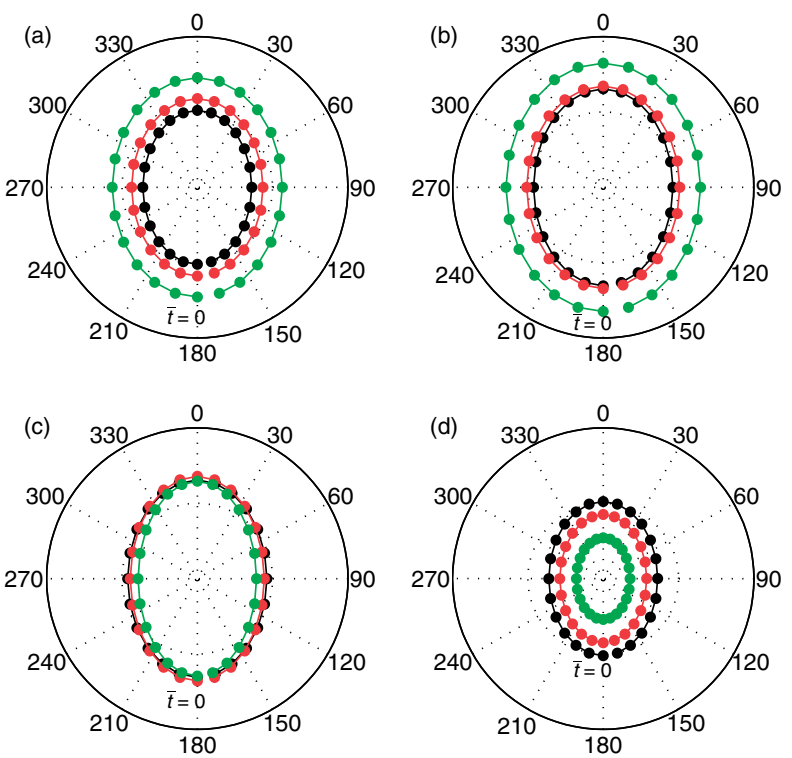

Figure 5. Near-surface wind perturbation hodograph $(\bar{z}=0.1)$ starting at $\bar{t}=0$, with data plotted every $2 \pi / 24$ time units (o marker), for $\bar{\Lambda}=0$ (black), 0.5 (red) and 0.9 (green) and for (a) $\bar{x}=-1.5$, (b) $\bar{x}=-0.5$, (c) $\bar{x}=0.5$ and (d) $\bar{x}=1.5$. In (a) and (d), the radius of the circle represents $\bar{u}=0.2$, whereas in (b) and (c), it represents $\bar{u}=0.4$.

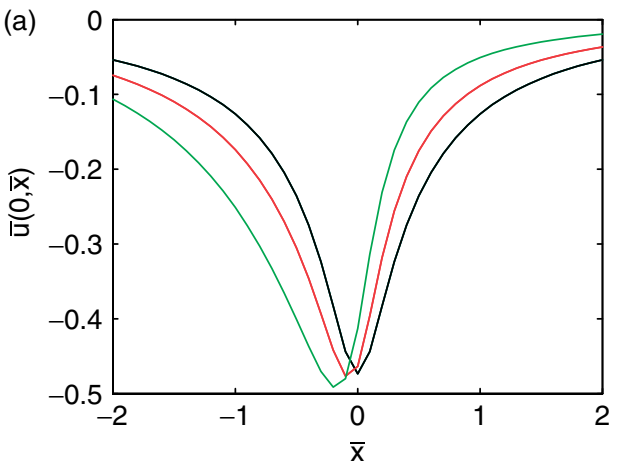

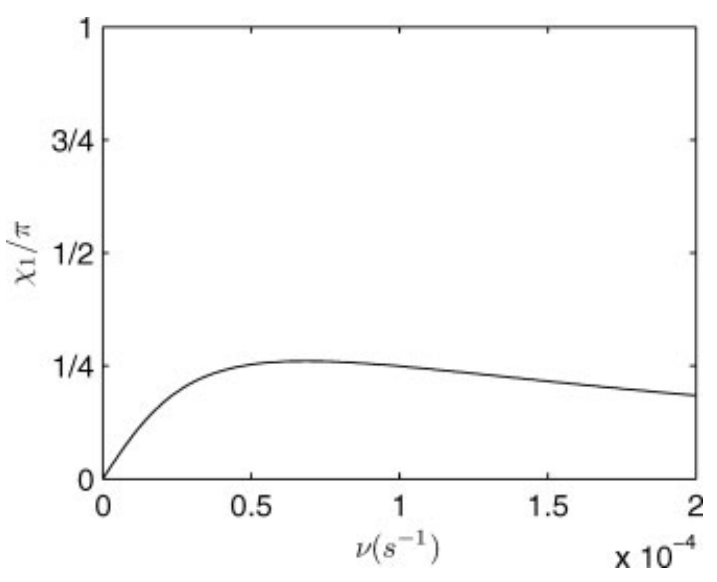

Figure 7. Phase lag $\chi_{1}$ of the Coriolis force relative to pressure gradient as a function of the linear friction parameter $v$ for $f=10^{-4} \mathrm{~s}^{-1}$.

by the along-shore jet has a tilt $O(\bar{\Lambda})$. When $\bar{\Lambda}=1$, the tilt of the breeze cell becomes equal to the slope of the background isentropes (which are in thermal wind balance with the wind shear). The critical slope of the sea-breeze tilt thus corresponds to the slope of the isentrope tilt. The present theory also predicts a spatial displacement between the heating pattern and the sea-breeze response, as the $u$ disturbance moves onshore with increasing the thermal wind shear $\bar{\Lambda}$.

The present linear theory is also used to interpret measurements made in the vicinity of New York City in the presence of a sustained synoptic southwesterly jet which occurs predominantly during the spring and summer (more than two events per month; CN10). There are very consistent results between the observations and the theory: the enhanced along-coast wind basically follows the tilted isentropes with a similar isentrope tilt, the clockwise-rotating wind hodograph showing the jet maximum peaking at 1800 LST (i.e. sunset at $\bar{t} \sim \pi$ ) is also predicted by the theory. The phase lag between the jet maximum at 1800 LST and the cross-shore pressure gradient maximum about $2-5 \mathrm{~h}$ earlier is less straightforward, but can be attributed to the angle made by the synoptic jet with respect to the coastline and/or the presence of friction. However, there is a need to quantify the contribution of the two processes, which is outside the scope of this study and is left for future work. The inland spatial shift of the breeze might also be indirectly confirmed by the coastal inland wind observations of larger diurnal amplitude for a stronger synoptic jet (thermal wind shear).

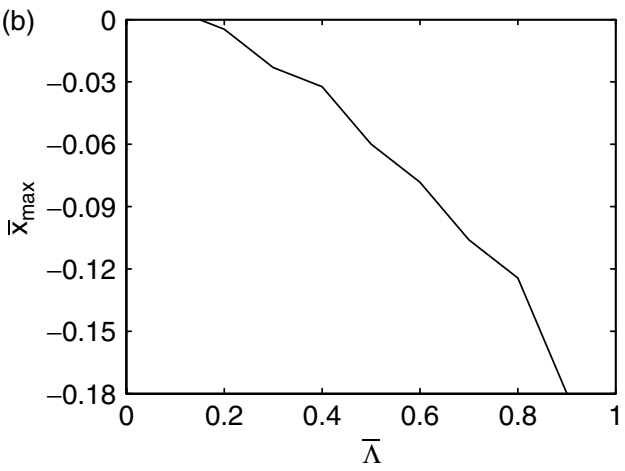

Figure 6. (a): Wind component $\bar{u}$ as a function of $\bar{x}$ for $\bar{\Lambda}=0$ (black), 0.5 (red) and 0.9 (green) at $\bar{t}=\pi / 2$. (b): Onshore distance $\bar{x}_{\text {max }}$ of the maximum wind speed $|\bar{u}|$ as a function of $\bar{\Lambda}$ at $\bar{t}=\pi / 2$. 
Finally, all the comparisons that can reasonably be made between such a simple model and a very complex reality show a broad agreement of some of the basic flow features. However a deeper modelling study, where the restrictions can be relaxed one at a time, is needed to include some key aspects of the cases observed in the 'real atmosphere' as, for instance, an explicit expression of the meridian pressure gradient. This is left for future work.

\section{Acknowledgements}

We are thankful to Brian Colle for fruitful discussions and to the two anonymous referees who helped to improve the manuscript significantly.

\section{References}

Baldi M, Dalu GA, Pielke Sr RA. 2008. Vertical velocities and available potential energy generated by landscape variability - Theory. J. Appl. Meteorol. Climatol. 47: 397-410.

Bastin S, Drobinski P. 2006. Sea breeze induced mass transport over complex terrain in southeastern France: a case study. Q. J. R. Meteorol. Soc. 132: 405-423.

Bastin S, Drobinski P, Dabas AM, Delville P, Reitebuch O, Werner C. 2005. Impact of the Rhône and Durance valleys on sea-breeze circulation in the Marseille area. Atmos. Res. 74: 303-328.

Bastin S, Drobinski P, Guénard V, Caccia JL, Campistron B, Dabas AM, Delville P, Reitebuch O, Werner C. 2006. On the interaction between sea breeze and summer mistral at the exit of the Rhône valley. Mon. Weather Rev. 134: 1647-1668.

Bluestein HB. 1993. Synoptic-Dynamic Meteorology in Midlatitudes. Vol. 2, Observations and Theory of Weather Systems. Oxford University Press: Oxford, UK.

Burk SD, Thompson WT. 1996. The summertime low-level jet and marine boundary layer structure along the California coast. Mon. Weather Rev. 124: 668-686.
Colle BA, Novak DR. 2010. The New York Bight jet: Climatology and dynamical evolution. Mon. Weather Rev. 138: 2385-2404.

Dalu GA, Pielke RA. 1989. An analytical study of the sea breeze. J. Atmos. Sci. 46: 1815-1825.

Drobinski P, Dubos T. 2009. Linear breeze scaling: From large-scale land/sea breezes to mesoscale inland breezes. Q. J. R. Meteorol. Soc. 135: $1766-1775$.

Drobinski P, Bastin S, Dabas AM, Delville P, Reitebuch O. 2006. Variability of the three-dimensional sea-breeze structure in southeastern France: observations and evaluation of empirical scaling laws. Ann. Geophys. 24: 1783-1799.

Drobinski P, Saïd F, Ancellet G, Arteta J, Augustin P, Bastin S, Brut A, Caccia JL, Campistron B, Cautenet S, Colette A, Coll I, Cros B, Corsmeier U, Dabas A, Delbarre H, Dufour A, Durand P, Guénard V, Hasel M, Kalthoff N, Kottmeier C, Lemonsu A, Lasri F, Lohou F, Masson V, Menut L, Moppert C, Peuch VH, Puygrenier V, Reitebuch O, Vautard R. 2007. Regional transport and dilution during high pollution episodes in southern France: Summary of findings from the ESCOMPTE experiment. J. Geophys. Res. 112: D13105, DOI: 10.1029/2006JD007494.

Estoque MA. 1962. The sea breeze as a function of the prevailing synoptic situation. J. Atmos. Sci. 19: 244-250.

Hakim GJ, Keyser D. 2001. Canonical frontal circulation patterns in terms of Green's functions for the Sawyer-Eliassen equation. Q. J. R. Meteorol. Soc. 127: 1795-1814.

Hoskins BJ, Bretherton FP. 1972. Atmospheric frontogenesis models: Mathematical formulation and solution. J. Atmos. Sci. 29: 11-37.

Morse PM, Feshbach H. 1953. Methods of Theoretical Physics. McGrawHill: New York.

Neumann J. 1984. The Coriolis force in relation to the sea and land breezes - A historical note. Bull. Amer. Meteorol. Soc. 65: 24-26.

Niino H. 1987. The linear theory of land and sea breeze circulation. J. Meteorol. Soc. Japan 65: 901-920.

Pielke RA. 1974. A three-dimensional numerical model of the sea breezes over south Florida. Mon. Weather Rev. 102: 115-139.

Qian T, Epifanio CC, Zhang F. 2009. Linear theory calculations for the sea breeze in a background wind: The equatorial case. J. Atmos. Sci. 66: $1749-1763$.

Rotunno R. 1983. On the linear theory of the land and sea breeze. J. Atmos. Sci. 40: 1999-2009.

Walsh JE. 1974. Sea-breeze theory and applications. J. Atmos. Sci. 31: 2012-2026 